\title{
Interaction of $\eta$-meson with light nuclei
}

\author{
V. B. Belyaev \\ Joint Institute for Nuclear Research, Dubna, 141980, Russia \\ S. A. Rakityansky*, S. A. Sofianos, and M. Braun \\ Physics Department, University of South Africa, P.O.Box 392, Pretoria 0001, South Africa \\ W. Sandhas \\ Physikalisches Institut, Universität Bonn, D-53115 Bonn, Germany
}

(November 13, 2018)

\begin{abstract}
A microscopic treatment of $\eta$-nucleus scattering is presented. When applying the underlying exact integral equations, the excitation of the target are neglected, and an input $\eta N$ amplitude is chosen which reproduces the $S_{11}(1535)$ resonance. It is shown that the $\eta$-nucleus scattering lengths are quite sensitive to the $\eta N$ parameters and the nuclear wave functions. For a special choice of the parameters an $\eta^{4} H e$ quasi-bound state occurs.
\end{abstract}

\section{INTRODUCTION}

There are at least three main reasons which motivate studies concerning $\eta$-nucleus systems.

The first one is of a fundamental character and is related to the possibility of studying the quark structure of $\eta$-mesons and the nucleon $S_{11}(1535)$-resonance, and the role played by the strange quarks in these systems. Recently, in experiments with $N \bar{N}$ annihilation into $\phi$ and $\omega$ channels [1], it was established that there is a strong polarization of the strange sea-quarks in nucleons. It is, therefore, interesting to investigate possible manifestation of this phenomenon also in $\eta$-nucleus systems.

The second reason concerns a pure nuclear problem, namely, the possible formation of $\eta$ - nucleus bound states. It was argued by Haider and Liu [2] that, due to the rather strong attraction in the $\eta N$-interaction at low energies, the $\eta$-meson, when immersed in the nucleus, might create a system which in some respects is analogous to a hypernucleus.

\footnotetext{
*Permanent address: Joint Institute for Nuclear Research, Dubna, 141980, Russia
} 
Estimations in [2] indicated that such a possibiltity exists for nuclei with atomic number $A \geq 12$.

Thirdly, Charge Symmetry Breaking (CSB) effects can be studied in processes involving $\eta$-production. Indeed, CSB was experimentally observed at Saturne [3] in the reaction

$$
d+d \longrightarrow{ }^{4} \mathrm{He}+\pi^{0}
$$

near the threshold of $\eta$-production. It was found that the production of pions was much greater than the CSB expected from electromagnetic interaction. The experimental crosssection is equal to $0.97 \mathrm{pb} / \mathrm{sr}$ (at $E_{d}=1100 \mathrm{MeV}$ ), while the one obtained from electromagnetic considerations is $0.003 \mathrm{pb} / \mathrm{sr}$ 近, the latter estimate being made at $600 \mathrm{MeV}$.

A possible explanation of CSB in the above reaction is that it can be attributed to the $\eta-\pi^{0}$ mixing, i.e., the process (1) can proceed via intermediate production of a pure $\eta^{0}$ meson state with isospin $I=0$,

$$
d+d \longrightarrow{ }^{4} H e+\eta^{0}
$$

which is an allowed reaction. The state $\left|\eta^{0}\right\rangle$ can be expressed as a linear combination of the physical ones, $|\eta\rangle$ and $\left|\pi^{0}\right\rangle$,

$$
\left|\eta^{0}\right\rangle=\frac{1}{\sqrt{1+\lambda^{2}}}\left(|\eta\rangle+\lambda\left|\pi^{0}\right\rangle\right)
$$

where the parameter $\lambda$ characterises the $\eta-\pi^{0}$ mixing. From Eq. (3) it follows that $\lambda$ is the proportionality constant between the amplitudes [5]

$$
f\left(d d \rightarrow \alpha \pi^{0}\right) \sim \lambda f(d d \rightarrow \alpha \eta) .
$$

The process (11), therefore, is no longer forbidden and one can expect a cross section much larger than the electromagnetic one.

Based on the above ideas, the authors of Ref. [6] obtained $0.12 \mathrm{pb} / \mathrm{sr}$ for the cross-section of the process (1) as compared to the experimental value of $0.97 \mathrm{pb} / \mathrm{sr}$. To explain this discrepancy, fully microscopic few-body calculations, which take into account the $\eta-\pi^{0}$ mixing, should be performed.

We would also like to mention the possibility of studying processes of the type

$$
d+d \longrightarrow{ }^{4} H e^{*}\left(\mathcal{J}^{P}, 0\right)+\pi^{0} .
$$

Here, ${ }^{4} H e^{*}\left(\mathcal{J}^{P}, 0\right)$ denotes excited states of ${ }^{4} H e$ nucleus with isotopic spin $I=0$ and different angular momenta and parities. These reactions might provide us with large cross sections due to the extended size of the excicted ${ }^{4} \mathrm{He}$ nucleus.

Having the above in mind, and in order to get some insight into the physics of $\eta$-nucleus systems, we undertook microscopic investigations concerning the low-energy behaviour of the $\eta$-nucleus elastic scattering amplitude and the positions of poles of the corresponding tmatrices in the complex $k$-plane. Another issue addressed is up to what extent the attraction of the two-body $\eta N$ interaction should be enhanced in order to produce quasi-bound states in the $\eta$-nucleus system. 


\section{THE METHOD}

Our method is based on the so-called Finite-Rank-Approximation (FRA) of the Hamiltonian proposed as an alternative to the multiple scattering theory of pion-nucleus interaction [7]. We, therefore, start by outlining this approach.

Consider the scattering of an $\eta$-meson from a nucleus of atomic number A. The total Hamiltonian of this system is

$$
H=H_{0}+V+H_{A}
$$

where $H_{0}$ is the kinetic energy operator (free Hamiltonian) of the $\eta$-nucleus motion , $V=$ $V_{1}+V_{2}+\cdots+V_{A}$ is the sum of $\eta N$-potentials, and $H_{A}$ is the total Hamiltonian of the nucleus. Introducing the Green function

$$
G_{A}(z)=\frac{1}{z-H_{0}-H_{A}},
$$

we obtain the following equation for the transition operator

$$
T(z)=V+V G_{A}(z) T(z) .
$$

For further developments, we introduce the (auxiliary) transition operator $T^{0}(z)$ via

$$
T^{0}(z)=V+V G_{0}(z) T^{0}(z)
$$

where

$$
G_{0}(z)=\frac{1}{z-H_{0}}
$$

is the free Green function. From the definition of $G_{0}(z)$ and $V$, it is clear that $T^{0}$ describes the scattering of mesons by a nucleus in which the position of the nucleons is fixed. The operator $T^{0}$ differs from the usual fixed center t-matrix in two respects. Firstly, Eq. (9) contains the kinetic energy operator $H_{0}$ describing the motion of the $\eta$-meson with respect to the center of mass of the target, secondly, the energy argument of $T^{0}$ is taken to be that of the total energy $z$ of the system.

Using the resolvent equation

$$
G_{A}(z)=G_{0}(z)+G_{0}(z) H_{A} G_{A}(z)
$$

we easily infer from (8) and (9)

$$
T(z)=T^{0}(z)+T^{0}(z) G_{0}(z) H_{A} G_{A}(z) T(z),
$$

a form suitable for the low-energy approximation on which our approach is essentially based. The spectral decomposition of the nuclear Hamiltonian $H_{A}$ reads

$$
H_{A}=\sum_{n} \mathcal{E}_{n}^{A}\left|\Psi_{n}^{A}\right\rangle\left\langle\Psi_{n}^{A}\left|+\int E\right| \Psi_{E}^{A}\right\rangle\left\langle\Psi_{E}^{A}\right| d E
$$


where $\left|\Psi_{n}^{A}\right\rangle$ are the bound-state eigenfuntions of $H_{A}$ with $\mathcal{E}_{n}$ being the correponding energies. Since we are interested in processes at energies far below the excited states or break-up thresholds of the nuclei, we can neglect all contributions to (13) except the ground-state part. That is, we use the approximation

$$
H_{A} \approx \mathcal{E}_{0}^{A}\left|\Psi_{0}^{A}><\Psi_{0}^{A}\right|
$$

Inserting Eq. (14) into Eq. (12) and sandwiching with $\left|\Psi_{0}^{A}\right\rangle$, we obtain the LippmannSchwinger-type equation

$$
T\left(\vec{k}^{\prime}, \vec{k} ; z\right)=T^{0}\left(\vec{k}^{\prime}, \vec{k} ; z\right)+\mathcal{E}_{0}^{A} \int \frac{d^{3} q}{(2 \pi)^{3}} \frac{T^{0}\left(\vec{k}^{\prime}, \vec{q} ; z\right)}{\left(z-\frac{q^{2}}{2 \mu}\right)\left(z-\mathcal{E}_{0}-\frac{q^{2}}{2 \mu}\right)} T(\vec{q}, \vec{k} ; z),
$$

where

$$
T^{0}\left(\vec{k}^{\prime}, \vec{k} ; z\right)=\int d^{3(A-1)} r\left|\Psi_{0}^{A}(\vec{r})\right|^{2} T^{0}\left(\overrightarrow{k^{\prime}}, \vec{k} ; \vec{r} ; z\right),
$$

Here $\vec{r}$ represents all nuclear Jacobi coordinates.

From a practical point of view it is convenient to rewrite Eq. (9) by using the Faddeevtype decomposition

$$
T^{0}(z)=\sum_{i=1}^{A} T_{i}^{0}(z)
$$

Introducing the operators

$$
t_{i}(z)=V_{i}+V_{i} \frac{1}{z-H_{0}} t_{i}(z)
$$

we finally get for the Faddeev components $T_{i}^{0}$ the following system of integral equations

$$
\begin{aligned}
T_{i}^{0}\left(\vec{k}^{\prime}, \vec{k} ; \vec{r} ; z\right) & =t_{i}\left(\vec{k}^{\prime}, \vec{k} ; \vec{r} ; z\right) \\
& +\int \frac{d^{3} k^{\prime \prime}}{(2 \pi)^{3}} \frac{t_{i}\left(\vec{k}^{\prime}, \vec{k}^{\prime \prime} ; \vec{r} ; z\right)}{z-\frac{k^{\prime 2}}{2 \mu}} \sum_{j \neq i} T_{j}^{0}\left(\vec{k}^{\prime \prime}, \vec{k} ; \vec{r} ; z\right) .
\end{aligned}
$$

The amplitude $t_{i}$ describes the scattering of the $\eta$-meson off the $i$-th nucleon. It is expressed in terms of the corresponding two-body $t_{\eta N}$-matrix via

$$
t_{i}\left(\vec{k}^{\prime}, \vec{k} ; \vec{r} ; z\right)=t_{\eta N}\left(\vec{k}^{\prime}, \vec{k} ; z\right) \exp \left[i\left(\vec{k}-\vec{k}^{\prime}\right) \cdot \vec{r}_{i}\right],
$$

where $\vec{r}_{i}$ is the vector from the nuclear center of mass to the $i$-th nucleon and can be expressed in terms of the Jacobi coordinates $\{\vec{r}\}$.

To get the nuclear wave function we used the so-called Integro-Differential EquationApproach (IDEA) 8,9]. In this approach the wave-function of a nucleus consisting of $\mathrm{A}$ nucleons is decomposed in Faddeev-type components

$$
\Psi(\mathbf{r})=\sum_{i<j \leq A} \psi_{i j}(\mathbf{r}),
$$


obeying

$$
\left(h_{0}-E\right) \psi_{i j}(\mathbf{r})=-V\left(r_{i j}\right) \sum_{k<l \leq A} \psi_{k l}(\mathbf{r}) .
$$

Here $h_{0}$ is the kinetic energy operator of the nucleus, and $V_{i j}$ is the potential between nucleons $i$ and $j$. These components are written as a product of a harmonic polynomial $H_{\left[L_{m}\right]}$ and an unknown function $P$

$$
\psi_{i j}(\mathbf{r})=H_{\left[L_{m}\right]}(\mathbf{r}) \frac{1}{\rho^{\mathcal{L}_{0}+1}} P(z, \rho),
$$

where $P$ depends only on the pair separation $r_{i j}=\sqrt{\rho(1+z) / 2}$ and the hyperradius $\rho=$ $\left[2 / A \sum r_{i j}^{2}\right]^{1 / 2}$. Eventually, one has to solve an integro-differential equation for this function $P$ which, for an A-boson system, reads [8.9]

$$
\begin{aligned}
\left\{\frac{\hbar^{2}}{m}[\right. & \left.-\frac{\partial^{2}}{\partial \rho^{2}}+\frac{\mathcal{L}_{0}\left(\mathcal{L}_{0}+1\right)}{\rho^{2}}-\frac{4}{\rho^{2}} \frac{1}{W_{0}(z)} \frac{\partial}{\partial z}\left(1-z^{2}\right) W_{0}(z) \frac{\partial}{\partial z}\right] \\
& \left.+\frac{A(A-1)}{2} V_{0}(\rho)-E\right\} P(z, \rho)= \\
& -\left[V\left(\rho \sqrt{\frac{1+z}{2}}\right)-V_{0}(\rho)\right]\left\{P(z, \rho)+\int_{-1}^{+1} f_{0}\left(z, z^{\prime}\right) P\left(z^{\prime}, \rho\right) d z^{\prime}\right\} .
\end{aligned}
$$

$V_{0}(\rho)$ is the hypercentral potential. The details of the method which takes into account the two-body correlations exactly can be found in Refs. [8, [9].

\section{RESULTS}

To solve Eq. (15) we need, as an input to Eq. (17), the two-body $\eta N$-amplitude (or the corrresponding t-matrix) and the ground state wave function $\Psi_{0}^{A}$ of the nucleus. For $t_{\eta N}$ we employ the $S$-wave separable form

$$
t_{\eta N}\left(k, k^{\prime}, z\right)=\frac{\lambda}{\left(k^{2}+\alpha^{2}\right)\left(z-E_{0}+i \Gamma / 2\right)\left(k^{2}+\alpha^{2}\right)}
$$

where $E_{0}$ and $\Gamma$ are the position and width of the $S_{11}$ resonance. Different sets of parameters $\alpha$ and $\lambda$ are chosen such that the scattering lengths in [10 are reproduced.

For such a form, the equation for $T^{0}$ can be solved analytically. The results thus obtained are given in Table 1. The required nuclear wave functions were constructed using the Integro-differential Equation with the Malfliet-Tjon I+III (MT) nucleon-nucleon potential [11]. As can be seen there is a strong dependence of the resulting $\eta$-nucleus scattering length on the two-body input. A large $\eta N$-scattering length generates a strong attraction of the $\eta$-meson by the three- and four-nucleon systems.

In Table 2 we present $\eta$-nucleus scattering lengths obtained by using two different sets of the nuclear wave functions, namely, the one constructed using the IDEA with MT potential 
and a phenomenological one of Gaussian form chosen to reproduce the root mean square radii of the nuclei obtained via the IDEA. We see that the considerable difference found for ${ }^{2} \mathrm{H}$ practically vanishes when going over to ${ }^{4} \mathrm{He}$. 


\section{TABLES}

TABLE I. The $\eta$-nucleus scattering lengths (in fm) for 9 combinations of the range parameter $\alpha$ and the $a_{\eta N}$ scattering lenght 10,12, 13.

\begin{tabular}{|c|c|c|c|c|}
\hline \hline \hline & $\alpha=2.357\left(\mathrm{fm}^{-1}\right)$ & $\alpha=3.316\left(\mathrm{fm}^{-1}\right)$ & $\alpha=7.617\left(\mathrm{fm}^{-1}\right)$ & $\alpha_{\eta N}(\mathrm{fm})$ \\
\hline${ }^{2} \mathrm{H}$ & $0.65+i 0.86$ & $0.62+i 0.91$ & $0.54+i 0.97$ & \\
${ }^{3} \mathrm{H}$ & $0.81+i 1.90$ & $0.66+i 1.98$ & $0.41+i 2.00$ & $0.27+i 0.22$ \\
${ }^{3} \mathrm{He}$ & $0.79+i 1.90$ & $0.64+i 1.98$ & $0.39+i 2.00$ & \\
${ }^{4} \mathrm{He}$ & $0.23+i 3.54$ & $-0.40+i 3.43$ & $-0.96+i 2.95$ & \\
\hline${ }^{2} \mathrm{H}$ & $0.74+i 0.77$ & $0.73+i 0.83$ & $0.67+i 0.91$ & \\
${ }^{3} \mathrm{H}$ & $1.12+i 1.82$ & $0.99+i 1.96$ & $0.71+i 2.07$ & $0.28+i 0.19$ \\
${ }^{3} \mathrm{He}$ & $1.10+i 1.83$ & $0.97+i 1.91$ & $0.68+i 2.07$ & \\
${ }^{4} \mathrm{He}$ & $0.96+i 3.99$ & $0.06+i 4.13$ & $-0.91+i 3.62$ & \\
\hline${ }^{2} \mathrm{H}$ & $1.38+i 2.27$ & $1.05+i 2.45$ & $0.57+i 2.34$ & \\
${ }^{3} \mathrm{H}$ & $-1.44+i 4.60$ & $-1.72+i 3.76$ & $-1.56+i 3.00$ & \\
${ }^{3} \mathrm{He}$ & $-1.42+i 4.54$ & $-1.68+i 3.74$ & $-1.53+i 2.99$ & \\
${ }^{4} \mathrm{He}$ & $-3.66+i 1.78$ & $-2.96+i 1.39$ & $-2.42+i 1.25$ & \\
\hline \hline
\end{tabular}

TABLE II. The $\eta$-nucleus scattering lengths (in fm) for different nuclear wave functions. In all cases $a_{\eta N}=0.55+i 0.30 \mathrm{fm}$.

\begin{tabular}{|c|c|c|}
\hline \hline \hline Model & IDEA & Gaussian \\
\hline \hline${ }^{2} \mathrm{H}$ & $1.05+i 2.45$ & $0.57+i 2.14$ \\
${ }^{3} \mathrm{H}$ & $-1.72+i 3.76$ & $-1.42+i 4.32$ \\
${ }^{3} \mathrm{He}$ & $-1.68+i 3.74$ & $-1.39+i 4.28$ \\
${ }^{4} \mathrm{He}$ & $-2.96+i 1.39$ & $-3.07+i 1.48$ \\
\hline \hline
\end{tabular}


To find at which values of the $\eta N$-scattering length a quasi-bound state appears in the $\eta$-nucleus system, we write the $\eta-N$ scattering length in terms of two parameters $g_{1}$ and $g_{2}$

$$
a_{\eta N}=\left(0.55 g_{1}+i 0.30 g_{2}\right) \mathrm{fm} .
$$

Note that $g_{1}=g_{2}=1$ is just one of the choices in Table 1 . These parameters are varied until the $\eta$-nucleus $T$-matrix exhibits a pole for $R e E=0$. Preliminary results of this search with Gaussian type wave functions are given in Table 3.

TABLE III. Values of $g_{1}$ at which a quasi-bound state appears.

\begin{tabular}{|c|r|r|r|r|}
\hline \hline \hline & $\alpha=2.357\left(\mathrm{fm}^{-1}\right)$ & $\alpha=3.316\left(\mathrm{fm}^{-1}\right)$ & $\alpha=7.617\left(\mathrm{fm}^{-1}\right)$ & $\mathrm{g}_{2}$ \\
\hline $\mathrm{d}$ & 1.610 & 1.613 & 1.547 & 0 \\
\cline { 2 - 5 } & 1.654 & 1.566 & 1.535 & 1 \\
\hline $\mathrm{t}$ & 1.293 & 1.124 & 0.996 & 0 \\
\cline { 2 - 5 } & 1.361 & 1.310 & 1.260 & 1 \\
\hline${ }^{3} \mathrm{He}$ & 1.302 & 1.204 & 1.130 & 1 \\
\cline { 2 - 6 } & 1.330 & 1.221 & 1.144 & 0 \\
\hline${ }^{4} \mathrm{He}$ & 1.075 & 0.992 & 0.910 & 1 \\
\cline { 2 - 6 } & 0.955 & 0.911 & 0.899 & 0 \\
\hline \hline
\end{tabular}

It is seen that an $\eta-{ }^{4} \mathrm{He}$ quasi-bound state for realistic values of $a_{\eta N}$ can exist. In all other cases, the physical $\eta N$ input, has to be modified in order to be able to support a corresponding quasi-bound state.

Finally, we mention that using the auxiliary matrix $T^{0}$ instead of the full solution of Eq. (15), we obtained completely different results.

\section{CONCLUSIONS}

We have investigated $\eta A$ systems with $A \leq 4$ in the framework of a few-body microscopic approach by using different two-body input parameters and nuclear bound state wave functions. The results can be summarized as follows:

- There is a remarkable increase of attraction with increasing atomic number.

- The same tendency appears for all nuclei when the $\eta N$ scattering length is increased.

- It is important in calculating $\eta A$ observables to use microscopically constructed wave functions.

- The fixed scatterer approximation, corresponding to the use of $T^{0}$ instead of $T$, is inadequate in describing the $\eta$-nucleus system.

- There is a strong indication that the $\eta^{4} H e$ system can form a quasi-bound state. 


\section{REFERENCES}

[1] M. G. Sapozhnikov, Preprint JINR (Dubna) E15-94-501; J. Ellis et al. CERN preprint, TH/94-7326, Geneva,1994.

[2] Q. Haider, L. C. Liu, Phys. Lett., 172 B, 257(1986)

[3] R. Frascaria et al., Phys. Rev. C 50, R537(1994)

[4] C. Y. Cheung, Phys. Lett., 119 B, 47(1982)

[5] C. Wilkin, Phys. Lett., 331 B, 276(1994)

[6] S. A. Coon and B. M. Preedons. Phys. Rev. C 33, 605(1986)

[7] V. B. Belyaev, in Lectures on the theory of few-body systems, Springer-Verlag, Heidelberg, 1990

[8] M. Fabre de la Ripelle, H. Fiedeldey, and S. A. Sofianos, Phys. Rev. C 38, 449(1988)

[9] W. Oehm, S. A. Sofianos, H. Fiedeldey, and M. Fabre de la Ripelle, Phys. Rev. C44, 81(1991)

[10] R. S.Bhalerao, L. C. Liu, Phys. Rev. Lett., 54, 865(1985)

[11] R. A. Malfliet and J.A. Tjon, Nucl. Phys. A127, 161 (1969); Ann. Phys. (N.Y.) 61, 425 (1970)

[12] C. Wilkin, Phys. Rev., C 47, R938(1993)

[13] C. Bennhold, H. Tanabe, Nucl. Phys., A 530, 625(1991) 